\title{
1.3GHZ INDUCTIVE OUTPUT TUBE FOR PARTICLE ACCELERATORS
}

\author{
A. Wheelhouse, E. Sobieradzki, e2v technologies, Chelmsford, Essex, UK
}

\begin{abstract}
There is an increasing requirement within the particle accelerator community for CW RF power sources in the L-band frequency range. The paper describes the development of a new inductive output tube (IOT) for use in high energy applications. A target specification of $16 \mathrm{~kW}$ CW output power at an efficiency of $60 \%$ was set and this paper presents results obtained to date. The tube uses an electron gun geometry that minimizes transit time effects in the cathode to grid gap and draws heavily on $\mathrm{e} 2 \mathrm{v}$ technologies' expertise in the design and manufacture of IOT's and klystrons.
\end{abstract}

\section{INTRODUCTION}

The IOT was invented in the 1930's, [1], however due to technological limitations it was not until the mid 1980's that the first high power IOTs, [2] were produced. The IOT is now an established technology that has become the standard high power RF amplifier tube throughout the terrestrial TV broadcast industry, with excellent life and reliability performance.

There are, at present, several synchrotron light source facilities being specified or built around the world that require high power RF amplifiers at $1.3 \mathrm{GHz}$. Having analysed the existing UHF $(470-860 \mathrm{MHz})$ IOT technology, it was clear that new design criteria were needed in order to build an IOT capable of operating at $1.3 \mathrm{GHz}$, while retaining the life, linearity, robustness and efficiency characteristics of established IOTs. The main issue identified was the possibility of transit time effects in the cathode to grid region of the electron gun that would otherwise reduce the efficiency of the IOT. In order to eliminate the transit time issue, it is necessary to design an electron gun with a significantly smaller cathode to grid gap. The gap must be mechanically stable throughout the range of operating temperatures that the IOT will experience, and that any warm-up transients must be limited to prevent cathode to grid short circuits.

The IOT produced by $22 \mathrm{v}$ technologies to address the $1.3 \mathrm{GHz}$ application has the following target specification:

$\begin{array}{ll}\text { Frequency } & 1.3 \mathrm{GHz} \\ \text { Output Power } & 16 \mathrm{~kW} \\ \text { Beam Voltage } & <28 \mathrm{kV} \\ \text { Efficiency } & >60 \% \\ \text { Gain } & >20 \mathrm{~dB} \\ \text { Class of operation } & \mathrm{B} \text { or AB }\end{array}$

\section{DESIGN}

Extensive computer modelling has been carried out to define the entire structure of the tube, but with a particular emphasis on the electron gun. The electron gun beam conditions were optimised in order to minimise transit time effects. The CW nature of the intended applications also led to the adoption of an integral output cavity / coupler system to optimise thermal and mechanical stability, whilst minimising the possibility of RF leakage. In order to validate the models at the prototype testing stage, initial prototypes were built with isolated collectors to allow body current measurements. Liquid cooling of the anode, inner conductor of the output line and output drift tube were also included. The inclusion of these features was also to allow validation of the models, and to assess the thermal stability and power dissipations of the areas found to be at greatest risk during modelling. It is anticipated that some or all of these additional arrangements will be eliminated from production devices. The prototype is shown in figure 1. For ease of visual scaling, the output waveguide is WR650.

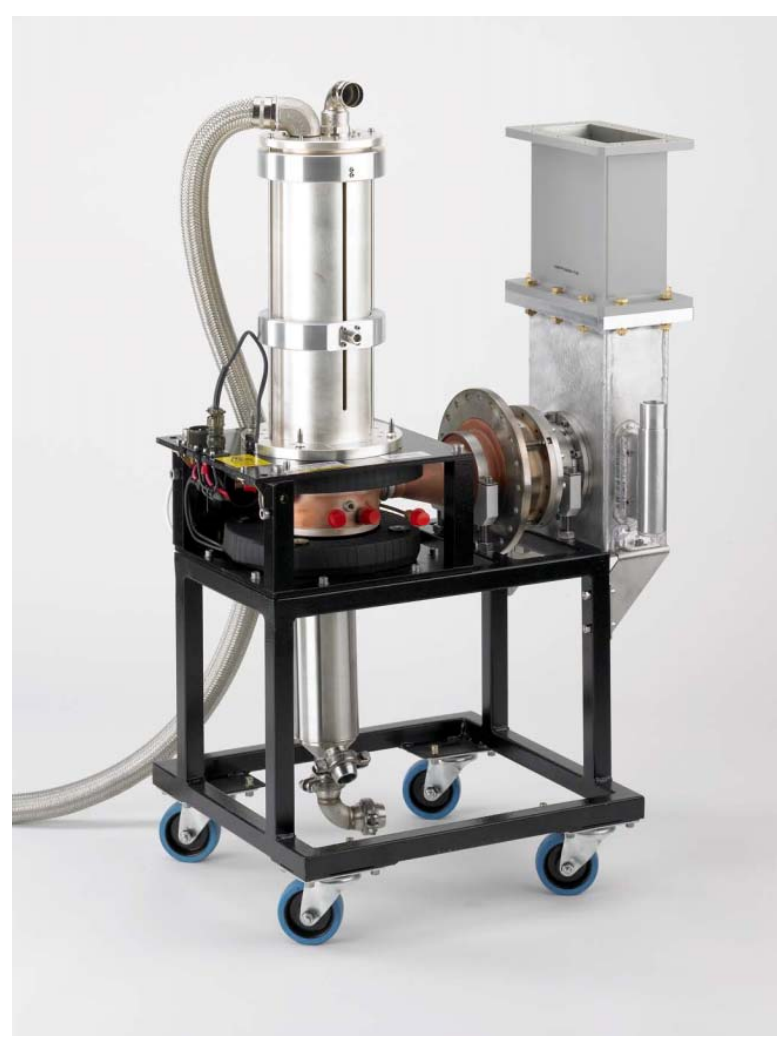

Figure 1: $1.3 \mathrm{GHz}$ IOT in its circuit.

Several modelling packages have been used to simulate the various aspects of the tube. The electron gun design was performed using OPERA-2d starting with the electrostatic beam optics. Once the performance was satisfactory, the design was refined with magnetic field 
applied. Analyses were performed at various operating conditions. Great care was taken to ensure a well-behaved beam was obtained. The coaxial input cavity was based on the cavity design used successfully in UHF television IOT's, and was optimised using MAFIA. The integral output cavity / coupler system was designed using both MAFIA and Microwave Studio. A radial button probe in the wall of the output cavity allows fine tuning of the frequency of the output cavity. The output from the IOT is fed to WR650 waveguide via a coaxial ceramic RF window and a coax to waveguide T-bar transition. The output gap and output drift tube were optimised using the 2D particle-in-cell module of MAFIA. This module was also used to determine the power densities on the inside of the collector. The collector dimensions were optimised using the CFD package FLUENT for $50 \%$ more power dissipation than nominal operating conditions.

ANSYS was used extensively to model the numerous critical thermal gradient and expansion issues that were identified as being potentially problematic. Care was taken in analysing the electron gun and in particular the cathode to grid region under numerous processes and operating conditions. The output from this modelling phase was then used to refine the mechanical design of the tube from its first conceptual layout to the final design that addressed all of the thermal expansion and stability issues.

\section{TEST RESULTS}

Test results taken from prototype tubes under $16 \mathrm{~kW}$ conditions are shown in Table 1. The results are for class $\mathrm{AB}$ operation and show that it has been possible to meet the target specification of efficiency and gain, even with a beam voltage as low as $25 \mathrm{kV}$. As is to be expected from a gridded device, the gain and efficiency of the tube can be traded off with each other by varying the quiescent current through altering the grid voltage.

Table 1: Test data for an output power of $16 \mathrm{~kW}$

\begin{tabular}{|c|c|c|c|c|c|}
\hline $\begin{array}{c}\text { Beam } \\
\text { Voltage } \\
(\mathrm{kV})\end{array}$ & $\begin{array}{c}\text { Beam } \\
\text { Current } \\
(\mathrm{A})\end{array}$ & $\begin{array}{c}\text { Grid } \\
\text { Voltage } \\
(\mathrm{V})\end{array}$ & $\begin{array}{c}\text { Drive } \\
\text { Power } \\
(\mathrm{W})\end{array}$ & $\begin{array}{c}\text { Gain } \\
(\mathrm{dB})\end{array}$ & $\begin{array}{c}\text { Efficiency } \\
(\%)\end{array}$ \\
\hline 25 & 1.03 & 80 & 140 & 20.6 & 62.1 \\
\hline 25 & 1.00 & 88 & 169 & 19.8 & 64.0 \\
\hline 26 & 0.97 & 91 & 152 & 20.2 & 63.4 \\
\hline 27 & 0.98 & 84 & 113 & 21.5 & 60.4 \\
\hline 27 & 0.92 & 96 & 158 & 20.1 & 64.4 \\
\hline 28 & 0.88 & 100 & 143 & 20.5 & 64.9 \\
\hline
\end{tabular}

For the results obtained the body current was below $20 \mathrm{~mA}$, and remained below $20 \mathrm{~mA}$ when the magnetic field focus current was altered over a 10A range, giving confidence in the modelling of the electron beam trajectories. Under these conditions the temperature differentials through the cooling circuits was found to remain constant.

Figure 2 shows the transfer curves for a prototype tube biased for grid voltages between $-75 \mathrm{~V}$ and $-90 \mathrm{~V}$. It can be seen that there is very little amplitude compression at the nominal operating power of $16 \mathrm{~kW}$, and that the tube is well away from saturation.

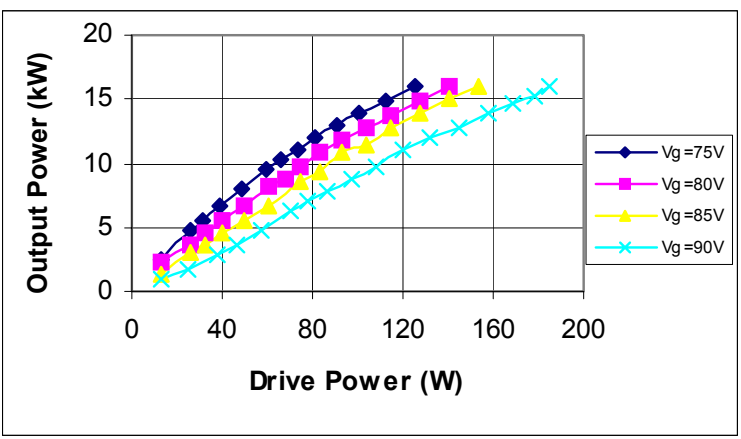

Figure 2: Transfer curves for a beam voltage of $25 \mathrm{kV}$.

Figure 3 shows the efficiency versus output power transfer curves for varying grid voltages. Efficiencies greater than $60 \%$ at $16 \mathrm{~kW}$ are achieved. However the efficiency is seen to increase with increasing grid voltage at the expense of gain (Figure 2).

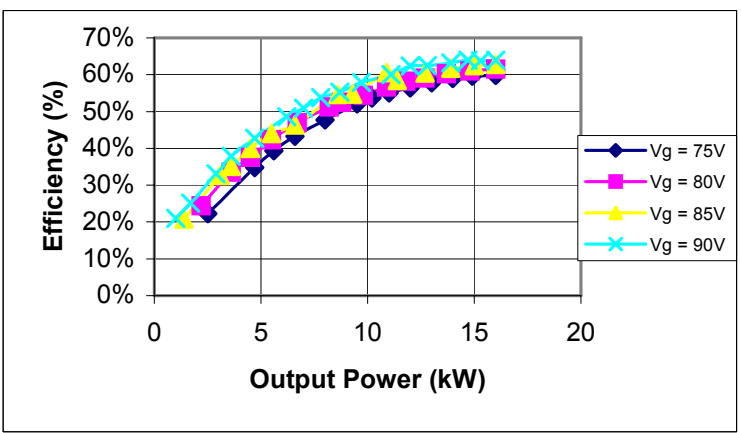

Figure 3: Efficiency versus output power at a beam voltage of $25 \mathrm{kV}$.

Thermal drift with power has been noted during testing and presently an analysis of these figures is under way so that improvements can be incorporated in future designs.

In parallel with data acquisition testing, prototype tubes have also been operated continuously at full power for extended periods to assess stability. Another tube has been left to cycle the electron gun structure thermally to simulate repeated cold starts. At the time of writing, neither of these tubes has shown any meaningful change in performance characteristics.

Further design improvements are currently under way to further improve the margin on the efficiency and the gain above the target specification. 


\section{SUMMARY}

The work done to date shows that it is possible to produce an IOT for operation at $1.3 \mathrm{GHz}$. The design with a reduced cathode to grid spacing has been shown to meet the target specification and is well aligned with computer modelling. Excellent linearity performance has been achieved, which makes the IOT suitable for operation as a high power RF amplifier in synchrotron light sources.

\section{REFERENCES}

[1] Andrew Haeff, Electronics, 12, 30, 1939

[2] Donald H. Preist, Merrald B. Shrader, "The Klystrode, an unusual transmitting tube with potential for UHFTV" proceedings of the IEEE, Vol. 70 No. 11 November 1982

\section{ACKNOWLEDGEMENT}

The authors express their thanks to their many colleagues, both within the $\mathrm{e} 2 \mathrm{v}$ technologies organisation and outside of it, for their valuable contributions to this paper. The ideas and opinions contained in the paper are attributable to the authors, and do not, necessarily reflect those of $2 \mathrm{v}$ technologies, or any associated companies. 